\section{(6) OPEN ACCESS}

\title{
An ankylosing spondylitis-associated genetic variant in the IL23R-IL12RB2 intergenic region modulates enhancer activity and is associated with increased Th1-cell differentiation
}

\author{
Amity R Roberts, ${ }^{1}$ Matteo Vecellio, ${ }^{1}$ Liye Chen, ${ }^{1}$ Anna Ridley, ${ }^{1}$ Adrian Cortes, ${ }^{2,3}$ \\ Julian C Knight, ${ }^{3}$ Paul Bowness, ${ }^{1}$ Carla J Cohen, ${ }^{1}$ B Paul Wordsworth ${ }^{1}$
}

\begin{abstract}
Handling editor Tore K Kvien
- Additional material is published online only. To view please visit the journal online (http://dx.doi.org/10.1136/ annrheumdis-2015-208640).

${ }^{1}$ Nuffield Department of Orthopaedics, Rheumatology and Musculoskeletal Sciences, Botnar Research Centre, University of Oxford, Oxford, UK

${ }^{2}$ Division of Clinical Neurology Nuffield Department of Clinical Neurosciences, John Radcliffe Hospital, University of Oxford, Oxford, UK

${ }^{3}$ Wellcome Trust Centre for Human Genetics, Roosevelt Drive, University of Oxford, Oxford, UK
\end{abstract}

\section{Correspondence to} Professor B Paul Wordsworth, Nuffield Department of Orthopaedics, Rheumatology and Musculoskeletal Sciences, Botnar Research Centre, Nuffield Orthopaedic Centre, Windmill Road, Headington, Oxford OX3 7LD, UK; paul. wordsworth@ndorms.ox.ac.uk

Received 22 September 2015 Accepted 2 February 2016 Published Online First 25 February 2016

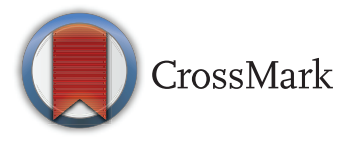

\footnotetext{
To cite: Roberts $A R$, Vecellio M, Chen L, et al. Ann Rheum Dis 2016;75:2150-2156.
}

\section{ABSTRACT}

Objectives To explore the functional basis for the association between ankylosing spondylitis (AS) and single-nucleotide polymorphisms (SNPS) in the IL23RIL 12RB2 intergenic region.

Methods We performed conditional analysis on genetic association data and used epigenetic data on chromatin remodelling and transcription factor (TF) binding to identify the primary AS-associated IL23R-IL 12RB2 intergenic SNP. Functional effects were tested in luciferase reporter assays in HEK293T cells and allelespecific TF binding was investigated by electrophoretic mobility gel shift assays. IL23R and IL $12 R B 2$ mRNA levels in CD4+ T cells were compared between cases homozygous for the AS-risk ' $A$ ' allele and the protective ' $G$ ' allele. The proportions of interleukin (IL)-17A+ and interferon (IFN)- $\gamma+$ CD4+ T-cells were measured by fluorescence-activated cell sorting and compared between these AS-risk and protective genotypes. Results Conditional analysis identified rs11209032 as the probable causal SNP within a $1.14 \mathrm{~kb}$ putative enhancer between IL23R and IL 12RB2. Reduced luciferase activity was seen for the risk allele $(p<0.001)$ and reduced $\mathrm{H} 3 \mathrm{~K} 4$ me1 methylation observed in $\mathrm{CD} 4+$ T-cells from 'A/A' homozygotes $(p=0.02)$. The binding of nuclear extract to the risk allele was decreased $\sim 3.5$-fold compared with the protective allele $(p<0.001)$. The proportion of IFN- $\gamma+$ CD4+ T-cells was increased in 'A/A' homozygotes ( $p=0.004)$, but neither IL23R nor IL 12RB2 mRNA was affected.

Conclusions The rs11209032 SNP downstream of IL23R forms part of an enhancer, allelic variation of which may influence Th1-cell numbers. Homozygosity for the risk ' $A$ ' allele is associated with more IFN- $\gamma$-secreting (Th1) cells. Further work is necessary to explain the mechanisms for these important observations.

\section{INTRODUCTION}

Ankylosing spondylitis (AS) is the prototypic spondyloarthropathy $(\mathrm{SpA})$, characterised by prominent axial skeletal involvement and enthesitis. ${ }^{1}$ Genome-wide association studies (GWAS) have clearly demonstrated the polygenic nature of AS. ${ }^{2-4}$ Further, many of the genes that have been implicated are also associated with conditions like inflammatory bowel disease (IBD) and psoriasis that occur much more commonly in individuals with AS than the general population. ${ }^{5}$ Of these,
IL23R (encoding the specific portion of the heterodimeric interleukin (IL)-23 receptor) was the first to be associated with AS. ${ }^{7}$ More than 40 loci have now been implicated in AS, several of which (eg, IL23R, IL12B, IL6R, TYK2, IL27R, IL1R2, IL1R1 and STAT3) potentially affect IL-23-driven pro-inflammatory pathways. ${ }^{2-4} 89$ The importance of these pathways is further highlighted by the potential role of IL23R-expressing cells at the entheses in murine models of SpA. ${ }^{10}$ The primary IL23R association with AS (also psoriasis and IBD) is with $r s 11209026$, a missense variant (Arg381Gln) in the cytoplasmic tail, which alters IL-23R signalling. 7112 In addition, a second independent association signal has been identified in the intergenic region downstream of $I L 23 R$ and upstream of IL12RB2 (encoding the $130 \mathrm{kD} \beta 2$ chain specific to the IL-12 receptor). ${ }^{3}$ This second signal is also associated with IBD. ${ }^{5}$ Currently, the mechanism underlying the latter association is unknown. In this study, we have identified a putative regulatory element (PRE) between IL23R and IL12RB2. We have then investigated the impact of AS-associated single-nucleotide polymorphisms (SNPs) on the function of this PRE.

\section{METHODS}

\section{Identification of a PRE}

We used a combination of published AS GWAS data $^{3}$ and epigenetic data from the ENCODE ${ }^{13}$ and Roadmap Epigenomics Projects, ${ }^{14}$ to identify a $1.14 \mathrm{~kb}$ PRE between IL23R and IL12RB2, including the AS-associated SNPs $r s 11209032$ and rs6677188. The epigenetic data included DNase I hypersensitivity sites, transcription factor (TF) binding sites and histone modifications.

\section{Patients with AS}

All patients in these studies fulfilled the modified New York AS criteria $^{15}$ or ASAS axial SpA imaging criteria. ${ }^{16}$ Following informed consent, blood samples for the functional studies (below) were obtained from patients.

\section{IFN- $\gamma+$ and IL-17A+ T-cell FACS analysis}

Blood samples were obtained from 52 biologicnaive AS cases (mean age 42 years \pm SD 12.3). The mean Bath AS disease activity index (BASDAI) was 4.6 \pm SD 2.2. 
Gene expression

Blood samples were obtained from 12 AS cases (mean age 61.5 years \pm SD 12.6). The mean BASDAI was $3.4( \pm S D$ 1.7) and mean $\mathrm{C}$ reactive protein $7.1 \mathrm{mg} / \mathrm{L}( \pm S D 6.4)$. Only nine were currently taking non-steroidal anti-inflammatory analgesics, and none were taking corticosteroids or other immunomodulatory drugs.

\section{Genotyping}

Historical typing data from previously published AS Immunochip study ${ }^{3}$ were used if available or were obtained using TaqMan Genotyping Assay (Life Technologies, Paisley, $\mathrm{UK})$ to assign SNP genotypes. Where required, DNA was extracted from peripheral blood mononuclear cells (PBMCs) using the QIAGEN AllPrep DNA/RNA Mini Kit (QIAGEN).

\section{CD4+ T-cell isolation}

CD4+ T-cells were isolated from PBMCs using the negative selection CD4+ T-cell Isolation kit (Miltenyi, Bisley, Surrey, UK). CD4+ T-cells were plated for $4 \mathrm{~h} /$ overnight in Roswell Park Memorial Institute supplemented with 10\% fetal bovine serum before harvesting for experiments. Cell viability was checked with trypan blue or fluorescence-activated cell sorting (FACS) analysis.

\section{FACS analysis}

PBMCs were isolated by density gradient centrifugation using Histopaque (Sigma, Dorset, UK), frozen and stored in liquid nitrogen before staining. Intracellular cytokine staining of Th17 and Th1-cells was carried out using BD Cytofix/Cytoperm kit (BD Bioscience, Oxford, UK). Cells were stimulated with $100 \mathrm{ng} / \mathrm{mL}$ phorbol 12-myristate 13-acetate (PMA) (Sigma, Dorset, UK) and $1 \mu \mathrm{g} / \mathrm{mL}$ ionomycin (Sigma, Dorset, UK) for $4 \mathrm{~h}$ in the presence of Golgi STOP and Golgi plug. After surface staining using CD3-BV605, CD4-APC and CD8-BV510 antibodies (Biolegend, London, UK), cells were fixed and permeabilised, then stained with IL-17A-FITC (eBiosciences, Ireland, UK) and interferon (IFN)- $\gamma$-AF700 (Biolegend). Dead cells were excluded using Fixable Viability Dye eFluor 780 (eBiosciences). Representative FACS plots of the gating strategy and intracellular staining are shown in online supplementary figure S1.

\section{Electrophoretic mobility shift assay}

Nuclear extract from HEK293 cells (human embryonic kidney cell line) was purchased from Active Motif (Belgium, Germany). Electrophoretic mobility shift assays (EMSAs) were performed with LightShift Chemiluminescent EMSA Kit (Thermo Scientific, Waltham, USA) using $5 \mu \mathrm{g}$ of nuclear extract, and $10 \mathrm{fmol}$ biotin labelled double-stranded oligonucleotides (50 bp fragment-Eurofins, Wolverhampton UK). The sequences of the synthetic single-stranded oligonucleotides used in the construction of these double-stranded oligonucleotides are listed in the online supplementary methods. Single-stranded biotinylated oligonucleotides were mixed and annealed at room temperature for $1 \mathrm{~h}$. Unlabelled competitor probes were in 100-fold excess. EMSAs were performed according to standard protocol (Thermo Scientific). The involvement of TWIST1 in these DNA-protein complexes was investigated by including TWIST1 antibody (ab50877-Abcam, Cambridge, UK). The results were confirmed in five independent experiments. Band intensity from these five experiments was measured by ImageJ Launcher (V.1.4.3.67). A detailed protocol is available in online supplementary methods.

\section{Luciferase reporter assay}

The $1.14 \mathrm{~kb}$ PRE sequence was amplified from genomic DNA and cloned into TA cloning kit pCR2.1 vector (Invitrogen, Paisley, UK). It was subcloned into pGL4.23 [luc2/minP] reporter vector (Promega, Madison, USA) at the SacI/XhoI restriction sites upstream of the minimal promoter necessary to drive the luciferase reporter gene (primer sequences available on request). Point mutations corresponding to genetic variants (G/A) of rs11209032 were introduced using the QuikChange II XL Site-Directed Mutagenesis Kit (Agilent, Santa Clara, USA). Luciferase reporter assay details are available in online supplementary methods.

\section{Quantitative real-time RT-PCR}

RNA was isolated from CD4+ T-cells from patients of different genotypes (PMA and ionomycin stimulated; overnight). RNA isolation was performed using the Allprep DNA/RNA Mini kit (QIAGEN) and cDNA synthesis (for $500 \mathrm{ng}$ RNA) was prepared with Superscript III from Invitrogen. A final concentration of $5 \mathrm{ng}$ was used in quantitative PCR (qPCR), which was performed with the ABI ViiA7 PCR instrument (Applied Biosystems, Paisley, UK) using SYBR Master mix (Applied Biosystems) with evaluation of dissociation curves. mRNA levels of each gene were quantified using the DDCt method and expressed relative to $\beta$-actin. For each gene, a TaqMan Gene Expression Assay was used (Life Technologies-according to the manufacturer's instructions); IL23R (Hs00332759_m1), IL12RB2 (Hs00155486_m1), IFNG (Hs00989291_m1), IL10 (Hs00961622_m1) and $\bar{\beta}$-actin (Hs01060665_g1). $\bar{H} 19$ primers were purchased from Diagenode (Liege, Belgium).

\section{Statistical analysis}

Association data for genotyped SNPs were obtained on the subset of AS cases of white British ancestry and white British controls from Immunochip GWAS. ${ }^{3}$ To evaluate the presence of independent effects on genetic susceptibility at the IL23RIL12RB2 intergenic region, we performed conditional analysis on 4230 AS cases and 9700 matched controls, as previously described. ${ }^{3}$ Association analysis was performed using the logistic regression function in PLINK, V.1.90, accounting for population structure with 10 principal components. ${ }^{17}$ One-way analysis of variance and two-tailed Student's t test were used to determine statistical significance using GraphPad Prism (V.5.03).

\section{RESULTS \\ Identification of a PRE within IL23R-IL12RB2 intergenic region associated with $A S$}

We identified a PRE (Chr1:67739940-67741075) located $14.2 \mathrm{~kb}$ downstream of IL23R and $33.1 \mathrm{~kb}$ upstream of IL12RB2 (figure 1). This PRE overlaps a region reported in the Immunochip study to be independently associated with AS after conditioning on association at the primary rs11209026 coding SNP. $^{3}$ This region is DNase I hypersensitive (in Th1-cells, but not Th17-cells) and exhibits TF binding (lymphoblastoid cell line; GM12878) and enhancer-associated H3K4me1 methylation (CD4+ CD25- IL-17A- T-cells; PMA and ionomycinstimulated). ${ }^{1} 314$

\section{Conditional analysis identifies rs 11209032 as a candidate casual variant}

The PRE contains 10 SNPs, 3 of which show strong or weak association with AS; $r s 11209032\left(\mathrm{p}=5.8 \times 10^{-16}\right), r s 80216366$ $\left(\mathrm{p}=4.9 \times 10^{-21}\right)$ and $r s 6677188 \quad\left(\mathrm{p}=2 \times 10^{-4}\right)$. Conditional 
Figure 1 Epigenetic and transcriptional landscape of the $1.14 \mathrm{~kb}$ putative regulatory element (PRE) containing $r s 11209032$ and rs6677188 downstream of IL23R. Cartoon representation of IL23R and IL12RB2 promoter and PRE location (Chr1:67739940-67741075). ENCODE and Roadmap data: (A) Transcription factor (TF) binding sites (grey/black box) in lymphoblastoid cell line GM12878. (B) DNase I hypersensitivity in Th17-cells. (C) H3K4me1 methylation in Th17-cells. (D) DNase I hypersensitivity in Th1-cells. (E) H3K4me1 methylation in Th1-cells. (F) Enhancer chromatin state in CD4+ CD25 - interleukin (IL)-17A- T-cells (PMA and ionomycin-stimulated).

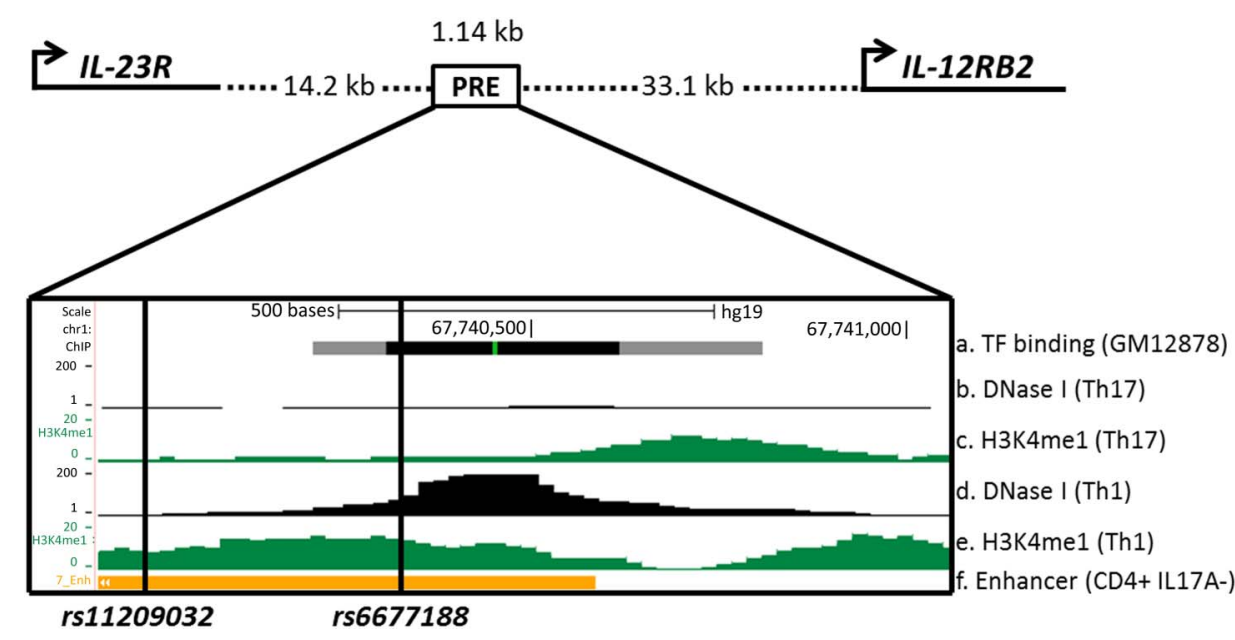

analysis previously performed on the primary IL23R coding SNP rs11209026 revealed no residual association at $r s 80216366(\mathrm{p}=0.4)$, indicating that this association is due to linkage disequilibrium (LD) with $r s 11209026 .^{3}$ The associations at $r s 11209032\left(\mathrm{p}=1.5 \times 10^{-9}\right)$ and $r s 6677188 \quad\left(\mathrm{p}=9.3 \times 10^{-7}\right)$ were robust to conditioning on $r s 11209026$, revealing a second region of independent association. ${ }^{3}$ We then performed further conditional analysis on $r s 11209032$ or rs6677188, respectively, and found that the association at rs6677188 disappeared after conditioning on $r s 11209032$, indicating that this association was due to LD with rs11209032 (table 1). In contrast, the strong association with $r s 11209032$ was retained $\left(\mathrm{p}=8.1 \times 10^{-12}\right)$ after conditioning on $r s 6677188$, thereby establishing the primacy of the rs11209032 association with AS in this region. However, a statistical association does not necessarily guarantee functionality. Initial interrogation of publicly available datasets suggested that $r s 6677188$ might actually be more functionally relevant than $r s 11209032$ (despite the genetic association data above). rs6677188 overlaps DNase I hypersensitivity (in Th1-cells) and TF binding sites (in GM12878 cells), whereas rs11209032 lies on the border of these sites (see figure 1). In the following functional study, we therefore analysed both SNPs.

\section{Homozygosity for the AS-risk allele is associated with increased Th1-cell frequencies}

We sought to determine the mechanisms by which $r s 11209032$ or rs6677188 affect AS susceptibility. The PRE containing rs11209032 and rs6677188 lies between IL23R (expressed on
Th17-cells) and IL12RB2 (expressed on Th1-cells). Therefore, we measured the frequencies of Th17- and Th1-cells from patients with known SNP genotypes. Th17-cell and Th1-cell frequencies were measured by FACS for IL-17A + and IFN- $\gamma+$ CD4+ T-cells, respectively (figure 2). A significant increase in the percentage of IFN- $\gamma+\mathrm{CD} 4+$ T-cells was observed in patients homozygous for the 'A' (risk) allele at $r s 11209032$ (figure $2 \mathrm{~A}, \mathrm{p}<0.01$ ), but not for IL-17A + CD4+ T-cells (figure $2 \mathrm{~B})$. The ' $\mathrm{T} / \mathrm{T}$ ' genotype at $r s 6677188$ is weakly associated with an increased percentage of IFN- $\gamma+$ CD4 + T-cells $(p=0.05$, figure 2C), but not IL-17A+ CD4+ T-cells (figure 2D). However, this observed increase in the percentage of IFN- $\gamma+$ CD4+ T-cells apparently associated with $r s 6677188$ is actually secondary to the association with the rs11209032 'A' (risk) allele, which is in complete $\mathrm{LD}$ with the $r s 6677188$ ' $\mathrm{T}$ ' allele. If the $r s 11209032$ 'A/A' homozygotes are excluded from the rs6677188 analysis (open circles in figure 2C), the apparent difference disappears. There was no correlation between the frequency of double-positive IL-17A+/IFN- $\gamma+$ CD4 + T-cells and the SNP genotype (see online supplementary figure S2). Overall, these results are consistent with the conditional analysis in suggesting that $r s 11209032$ is the most probable causal variant (through effects on Th1-cell differentiation). Support for an effect in Th1-cells comes from publicly available data sets that show the presence of DNase I hypersensitivity in Th1-cells within the PRE, but not in Th17-cells (see figure 1). Consequently, all the subsequent experiments focused on rs11209032 alone.

\begin{tabular}{|c|c|c|c|c|c|c|c|}
\hline Position* & Conditional SNP & Risk/protective & SNP & $\mathrm{p}$ Value & OR & RAF (case/control) & LD $\left(r^{2} / D^{\prime}\right)$ with conditional SNP \\
\hline \multirow[t]{3}{*}{ Chr1:67706208 } & $r s 11209026 \dagger$ & $\mathrm{G} / \mathrm{A}$ & rs11209032 & $1.5 \times 10^{-9}$ & 1.17 & $0.96 / 0.93$ & $0.03 / 0.97$ \\
\hline & & & rs80216366 & 0.4 & 1.11 & & $0.83 / 0.94$ \\
\hline & & & rs6677188 & $9.3 \times 10^{-7}$ & 0.91 & & $0.01 / 0.87$ \\
\hline \multirow[t]{3}{*}{ Chr1:67740342 } & rs11209032 & $A / G$ & rs11209026 & $9.5 \times 10^{-14}$ & 0.62 & $0.37 / 0.33$ & $0.03 / 0.97$ \\
\hline & & & rs80216366 & $3.6 \times 10^{-10}$ & 0.69 & & $0.03 / 0.98$ \\
\hline & & & rs6677188 & 0.2 & 1.04 & & $0.17 / 1$ \\
\hline \multirow[t]{3}{*}{ Chr1:67740653 } & $r s 6677188$ & $\mathrm{~A} / \mathrm{T}$ & rs11209026 & $3 \times 10^{-18}$ & 0.58 & $0.24 / 0.25$ & $0.01 / 0.87$ \\
\hline & & & rs11209032 & $8.1 \times 10^{-12}$ & 1.23 & & $0.17 / 1$ \\
\hline & & & rs80216366 & $1.3 \times 10^{-14}$ & 0.63 & & $0.02 / 1$ \\
\hline
\end{tabular}

${ }^{*} \mathrm{NCBI}$ Build 37 human genome coordinates.

tData from ref. 3

Chr., chromosome; LD, linkage disequilibrium; RAF, risk allele frequency. 
Figure 2 Homozygosity for the ankylosing spondylitis (AS)-risk allele at $r$ 111209032 is associated with increased Th1-cell frequencies. (A) The $\%$ of interferon (IFN)- $\gamma+$ CD4+ T-cells in patients with $A S$ of each genotype at $r s 11209032$ (19 GG, $25 \mathrm{GA}, 8 \mathrm{AA})$. (B) The $\%$ of interleukin (IL)-17A+ CD4 + T-cells in patients with AS of each genotype at $r s 11209032$ (19 GG, 25 GA, 8 AA). (C) The \% of IFN- $\gamma+$ CD4+ $\mathrm{T}$-cells in patients with AS of each genotype at rs6677188 (33 TT, 17 TA, 2 AA). Patients 'A/A' at rs 11209032 are highlighted by open circles. (D) The $\%$ of IL-17A+ CD4+ T-cells in patients with AS of each genotype at rs6677188 (33 TT, 17 TA, 2 AA). The percentage of cells is expressed as mean \pm SEM. Student's t test was used.
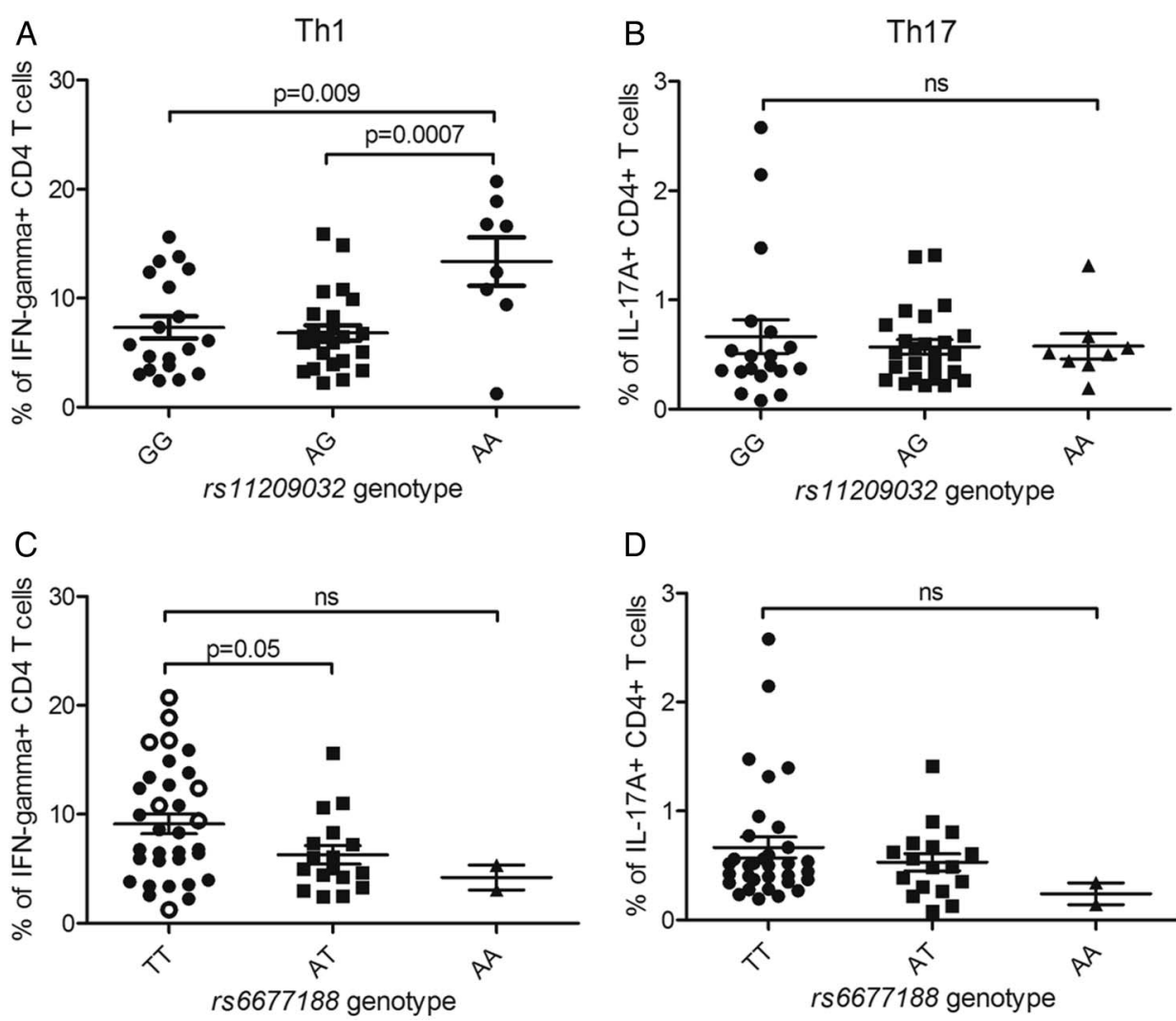

\section{Differential binding of nuclear extract at rs11209032}

We investigated the mechanism for the increase in IFN- $\gamma+$ CD4+ T-cells (above) by looking for effects of $r s 11209032$ on TF binding. EMSAs were performed using nuclear extract from HEK293 cells (human embryonic kidney cell line). The addition of nuclear extract to a 50mer DNA probe containing rs11209032 revealed a DNA-protein complex (i), with $\sim 3.5$-fold lower binding to the 'A' (risk) allele (figure 3,

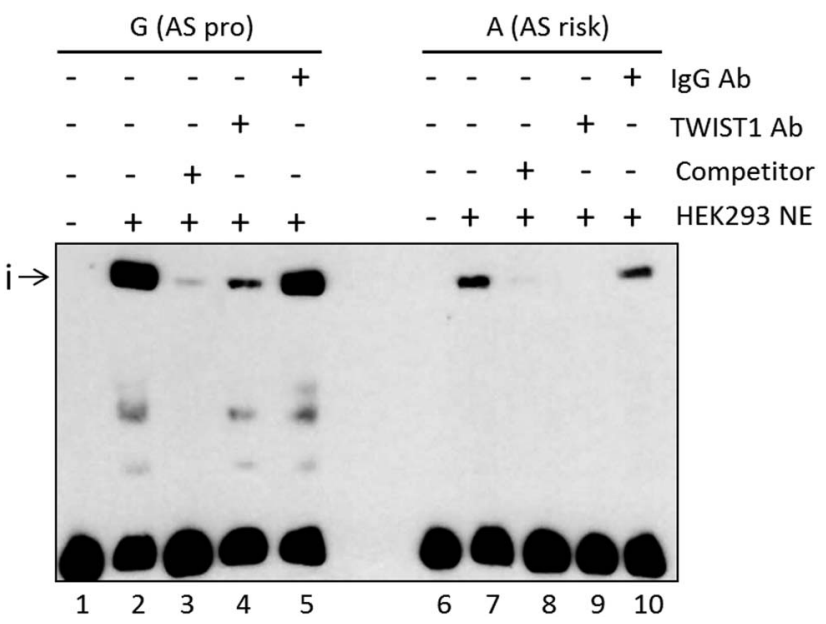

Figure 3 The ankylosing spondylitis (AS)-risk allele at $r s 11209032$ alters DNA-protein complex formation. Chemiluminescent electrophoretic mobility shift assay (EMSA) showing complex formation (i) after addition of HEK293 nuclear extract (lanes 2 and 7), and competition with 100-fold excess of unlabelled probes (lanes 3 and 8). TWIST1 antibody addition leads to a reduction or inhibition of the complex (i) (lanes 4 and 9, respectively). Addition of nonspecific IgG antibody (lanes 5 and 10). NE, nuclear extract; pro, protective. This result was confirmed in five independent experiments. $\mathrm{p}<0.001$ ). Binding to the ' $\mathrm{G}$ ' (protective) allele was outcompeted by 100 -fold excess of unlabelled ' $G$ ' (figure 3 and online supplementary figure S3A).

A search for TF binding consensus sequences that directly overlap rs11209032 revealed a CTCF binding motif (in lymphoblastoid cell line GM12878). ${ }^{18}$ However, rs11209032 genotype had no influence on binding (data not shown). The consensus sequence of the Th1 transcriptional repressor TWIST1 $\left(5^{\prime}\right.$-NCANNTGN-3') is located 1 bp $3^{\prime}$ of the $r s 11209032$ SNP. HEK293 cells are a good source of TWIST1 protein (see online supplementary figure S3B). Addition of TWIST1 antibody to the EMSA reduced/inhibited the formation of the DNA-protein complex for both alleles (figure 3).

\section{rs11209032 alters levels of H3K4me1 methylation}

Publicly available data show that the region overlapping rs11209032 is enriched for enhancer-associated H3K4me1 methylation in Th1-cells. ${ }^{14}$ To investigate enhancer activity ex vivo, we assessed the levels of H3K4me1 methylation at rs11209032 by ChIP-qPCR. Homozygosity for the 'A' (risk) allele correlated with reduced $\mathrm{H} 3 \mathrm{~K} 4 \mathrm{me} 1$ abundance in CD4+ T cells from patients with AS, whereas the level in ' $G / G$ ' homozygotes was similar to the positive control (IL10 enhancer) (figure 4A, $\mathrm{p}=0.02$ ).

\section{The AS-risk allele at rs11209032 shows reduced reporter activity}

We used luciferase reporter assays to confirm the effect of rs11209032 genotype on enhancer activity. Luciferase reporter assays were performed in HEK293T cells transfected with pGL4.23 plasmids containing the minimal promoter ( $\mathrm{minP})$ and the $1.14 \mathrm{~kb}$ PRE sequence (see figure 1) with either the 'A' (risk) allele or the ' $G$ ' (protective) allele. The 'G' (protective) allele showed significantly increased reporter activity above the minP 
Figure 4 Homozygosity for the ankylosing spondylitis (AS)-risk allele at rs11209032 is associated with reduced enhancer activity without altering mRNA levels of IL23R or IL12RB2. (A) H3K4Me1 methylation ChIP-qPCR assessed at the $r s 11209032$ locus in CD4+ T cells (PMA and ionomycin-stimulated) from three ' $G / G$ ' and three 'A/A' patients. The fold enrichment is expressed as mean \pm SEM for each patient in triplicate. Positive control is IL10 enhancer. Student's t test was used. (B) The transcriptional activity of $r s 11209032$ compared with $\min P$ (set to 1) was measured by luciferase reporter assays in HEK293T cells. The values of relative luciferase activity are expressed as mean \pm SEM of three or four repeat experiments each done in triplicate. One-way analysis of variance was used. (C) Relative amount of IL23R mRNA in primary CD4+ T-cells (PMA and ionomycin-stimulated) from $6 \mathrm{GG}$ and 6 AA patients (normalised against $\beta$-actin). mRNA levels are expressed as mean \pm SEM. Student's t test was used. (D) Relative amount of IL12RB2 mRNA in primary CD4+ T-cells (PMA and ionomycin-stimulated) from $6 \mathrm{GG}$ and 6 AA patients (normalised against $\beta$-actin). mRNA levels are expressed as mean \pm SEM. Student's $t$ test was used. minP, minimal promoter.
A

H3K4me1
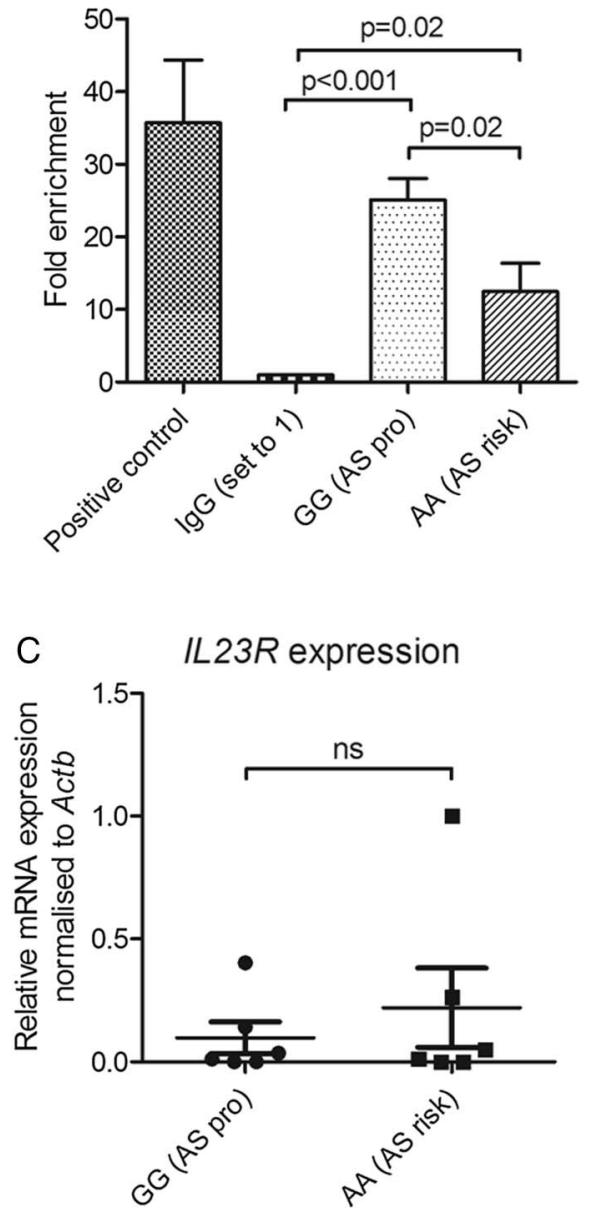

B

HEK293T cells
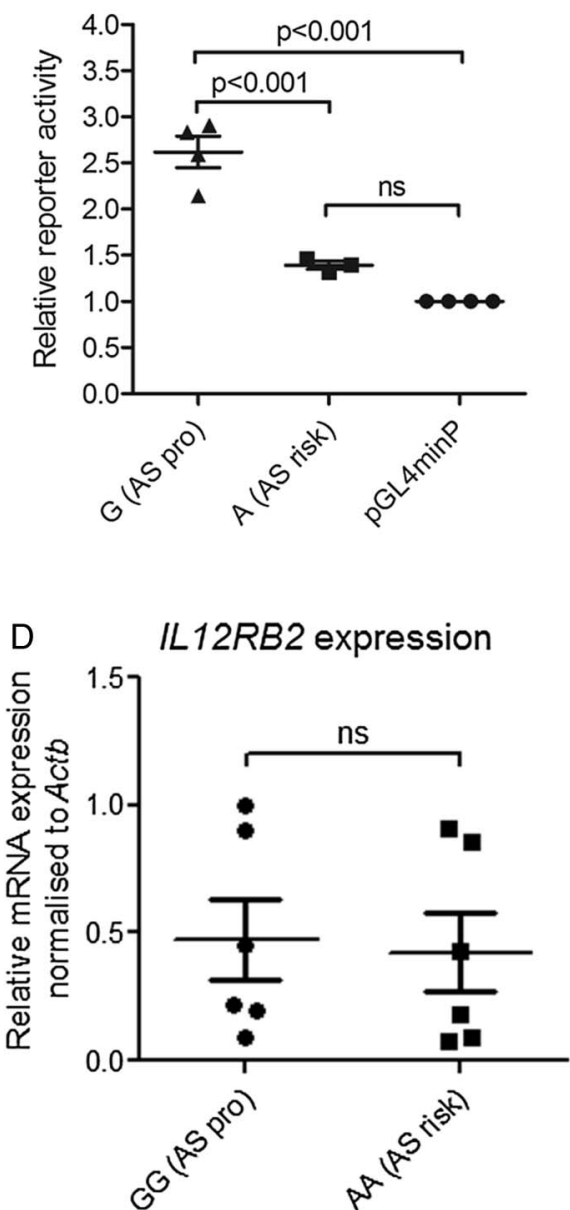

level of 1 (figure 4B, p<0.001), which provides further support to PRE having enhancer activity. Interestingly, the 'A' (risk) allele significantly reduced this activity compared with the protective allele $(\mathrm{p}<0.001)$.

\section{No correlation between rs11209032 genotype and IL23R or IL12RB2 mRNA levels}

We next assessed the effect of the rs11209032 genotype and reduced enhancer activity on expression levels of the nearby genes. Analysis of IL23R and IL12RB2 expression in CD4+ T-cells isolated from patients with AS (PMA and ionomycinstimulated overnight, cell viability $\geq 71 \%$ ) with different rs11209032 genotypes (6 GG and 6 AA) showed no significant effect on mRNA levels (figure 4C,D). There was also no correlation between disease activity and mRNA levels for either gene (data not shown).

\section{DISCUSSION}

We have clearly shown the primacy of the $r$ s11209032 SNP in a potentially important regulatory region between IL23R and IL12RB2 associated with AS. The $r s 11209032$ AS-risk 'A' allele influences the formation of a TF complex, which includes TWIST1. Luciferase reporter assays showed an effect of the AS-risk allele on transcription but, somewhat surprisingly, we could not detect an effect on expression of the two nearby genes $I L 23 R$ or $I L 12 R B 2$. Our experiments on gene expression were not highly powered and the absence of definitive high and/ or low expresser cell type controls somewhat hindered their interpretation. Overall, these data suggest that this regulatory element does not influence the expression of the neighbouring genes. However, we have not formally excluded the possibility it could modulate the function of these genes under different stimulation conditions from those that we have used or according to the differentiation status of the cells. It is also possible that there may be other regulatory elements in this region, which we have not yet identified that could influence these genes. These possibilities will be investigated systematically in future studies.

AS is a complex polygenic disease showing multiple genetic associations with the IL-23 pathway. Here, we show that homozygosity for the AS-risk ' $A$ ' allele of an SNP in the $I L-23 R-I L 12 R B 2$ intergenic region is associated with increased Th1-cell numbers. We have not been able to define the regulatory mechanism(s) involved precisely. Our EMSA data show that TWIST1 binds to the region containing $r s 11209032$. TWIST1 is reported to be a transcriptional repressor of Th1 gene expression and cytokine production, specifically reducing If $n-\gamma$ expression in mouse cells. ${ }^{19}$ It is, therefore, possible that altered binding of TWIST1 to the putative enhancer may contribute to the findings reported here. We now intend to investigate more deeply the involvement of TWIST1 and the full nature of the DNA-protein complex with rs11209032, which is reduced in the presence of the risk 'A' allele, to look for a potential network of genes that might be regulated at least in part by this SNP.

Inspection of $2 \mathrm{Mb}$ of flanking sequence around rs11209032 reveals no obvious Th1-related genes. However, since only $27 \%$ of distal regulatory elements are reported to interact with the 
nearest promoter, the target of an SNP disease association is often not with the nearest gene and may extend over several megabases. $^{20} 21$ Substantial work may be required to identify the target gene(s).

Double-positive IL-17A+/IFN- $\gamma+$ CD4 + T-cells have been reported in synovial fluid and synovial tissue of some patients with rheumatoid arthritis (RA) ${ }^{22-24}$ and are increased in peripheral blood from patients with AS and RA. ${ }^{25}$ However, we found no evidence of an influence of SNP genotype on the frequency of these cells. Previously, increased IFN- $\gamma$ production has been reported in T-cells from patients with $\mathrm{AS},{ }^{26-28}$ although this is controversial because others report reduced IFN- $\gamma$ levels. ${ }^{29}$ Here, we report a correlation of $r s 11209032$ genotype with the percentage of IFN- $\gamma+$ CD4 + T-cells in patients with AS. Our results highlight the potential importance of genetic/epigenetic regulation of the Th1 pathway in the pathogenesis of AS.

There are at least two distinct genetic effects arising from the vicinity of IL23R in AS. The first ( $r$ 11209026) has been previously characterised and encodes an Arg381Gln change in the cytoplasmic tail of IL-23R that reduces signalling both in healthy donors and patients with inflammatory arthritis. ${ }^{11} 123031$ Such protein-coding changes constitute only a minority of genetic associations with common diseases, perhaps indicative of their relatively pronounced functional effects. In contrast, more subtle effects on gene expression or protein binding, as represented by the IL23R-IL12RB2 association we describe here, are probably more common and may act synergistically. ${ }^{32}$ Our study highlights the power of conditional analysis to identify the primary genetic disease associations definitively. On first inspection, rs6677188, which lies in the same associated region as rs11209032, appears somewhat more likely to be functionally relevant because it is closer to the local peak of DNase I hypersensitivity. However, the conditional analysis unequivocally demonstrated the primacy of the rs11209032 association since the apparent association with rs6677188 disappeared after conditioning on $r s 11209032$.

Genetic associations with disease identified by GWAS can potentially identify new drug targets even where the strength of the association is relatively weak. In general, polymorphisms influencing gene expression are more likely to be implicated in polygenic diseases like AS. Additive influences arising from numerous SNPs in the IL-23 pathway, which alter the effector functions of Th1-cells and Th17-cells in patients with SpA, have been described ${ }^{32}$ but the full complexity of the regulation of these cells is only just becoming apparent. ${ }^{33}$

The combination of an analytical approach with a computational and experimental validation can identify important transcriptional gene networks, such as those governing the specification and function of Th1-cells in health and disease (eg, T-bet, STAT4, RUNX3 and TWIST1). ${ }^{19}{ }^{34} \mathrm{~A}$ similar approach has been used to identify and explain the association between a functional polymorphism in the tumour necrosis factor receptor gene (TNFRSF1A) and multiple sclerosis. ${ }^{35}$ Further studies in AS will focus on identifying the factors contributing to the reduced enhancer activity reported here and how these factors contribute to increased Th1-cell signalling.

Acknowledgements The authors wish to thank all the study participants who generously donated their DNA and peripheral blood to this study. The authors also thank Louise Appleton for DNA isolation of patient samples.

Contributors BPW and CJC are joint senior authors. ARR, MV, LC, AR, AC, JCK, PB, CJC and BPW: conceived and designed the experiments. ARR, LC, MV, AR and CJC: performed the experiments. ARR, MV, LC, PB, CJC and BPW: analysed the data. ARR, MV, LC, AC, JCK, PB, CJC and BPW: wrote the manuscript.
Funding ARR was funded by Arthritis Research UK (grant 20402), MV by National Institute for Health Research (NIHR) Oxford comprehensive Biomedical Research Centre (immunity and inflammation theme A93081), LC by Arthritis Research UK (grant 20235) and AR by NIHR Oxford Comprehensive Biomedical Research Centre. JCK is funded by the European Research Council under the European Union's Seventh Framework Programme (FP7/2007-2013)/ERC Grant agreement no. 281824, Arthritis Research UK (grant 20773) and the NIHR Oxford Comprehensive Biomedical Research Centre. Additional funding was provided by Arthritis Research UK (grants 19356, 18797 and 20796), the NIHR Thames Valley collaborative research network, NIHR Oxford Musculoskeletal Biomedical Research Unit and National Ankylosing Spondylitis Society (UK)

Competing interests None declared.

Ethics approval Oxford C Research Ethics Committee 06/Q1606/139 and Oxford B Research Ethics Committee 07/Q1605/35.

Provenance and peer review Not commissioned; externally peer reviewed.

Open Access This is an Open Access article distributed in accordance with the terms of the Creative Commons Attribution (CC BY 4.0) license, which permits others to distribute, remix, adapt and build upon this work, for commercial use, provided the original work is properly cited. See: http://creativecommons.org/ licenses/by/4.0/

\section{REFERENCES}

1 Dougados M, van der Linden S, Juhlin R, et al. The European Spondylarthropathy Study Group preliminary criteria for the classification of spondylarthropathy. Arthritis Rheum 1991;34:1218-27.

2 Evans DM, Spencer CCA, Pointon JJ, et al. Interaction between ERAP1 and HLA-B27 in ankylosing spondylitis implicates peptide handling in the mechanism for HLA-B27 in disease susceptibility. Nat Genet 2011:43:761-7.

3 Cortes A, Hadler J, Pointon JP, et al. Identification of multiple risk variants for ankylosing spondylitis through high-density genotyping of immune-related loci. Nat Genet 2013;45:730-8.

4 Reveille JD, Sims AM, Danoy P, et al. Genome-wide association study of ankylosing spondylitis identifies non-MHC susceptibility loci. Nat Genet 2010;42:123-7.

5 Duerr RH, Taylor KD, Brant SR, et al. A genome-wide association study identifies IL23R as an inflammatory bowel disease gene. Science 2006;314:1461-3.

6 Liu Y, Helms C, Liao W, et al. A genome-wide association study of psoriasis and psoriatic arthritis identifies new disease loci. PLoS Genet 2008;4:e1000041.

7 Burton PR, Clayton DG, Cardon LR, et al. Association scan of 14500 nonsynonymous SNPs in four diseases identifies autoimmunity variants. Nat Genet 2007;39:1329-37.

8 Karaderi T, Harvey D, Farrar C, et al. Association between the interleukin 23 receptor and ankylosing spondylitis is confirmed by a new UK case-control study and meta-analysis of published series. Rheumatology (Oxford) 2009:48:386-9.

9 Brown MA, Kenna T, Wordsworth BP. Genetics of ankylosing spondylitis-insights into pathogenesis. Nat Rev Rheumatol 2016;12:81-91.

10 Sherlock JP, Joyce-Shaikh B, Turner SP, et al. IL-23 induces spondyloarthropathy by acting on ROR- $\gamma \mathrm{t}+\mathrm{CD} 3+\mathrm{CD} 4-\mathrm{CD} 8-$ entheseal resident T cells. Nat Med 2012;18:1069-76.

11 Di Meglio P, Di Cesare A, Laggner U, et al. The IL23R R381Q gene variant protects against immune-mediated diseases by impairing IL-23-induced Th17 effector response in humans. PLOS ONE 2011;6:e17160.

12 Di Meglio P, Villanova F, Napolitano L, et al. The IL23R A/GIn381 allele promotes IL-23 unresponsiveness in human memory T helper 17 cells and impairs Th17 responses in psoriasis patients. J Invest Dermatol 2013;133:2381-9.

13 ENCODE Project Consortium. An integrated encyclopedia of DNA elements in the human genome. Nature 2012;489:57-74.

14 Bernstein BE, Stamatoyannopoulos JA, Costello JF, et al. The NIH Roadmap Epigenomics Mapping Consortium. Nat Biotech 2010;28:1045-8.

15 van der Linden S, Valkenburg HA, Cats A. Evaluation of diagnostic criteria for ankylosing spondylitis. A proposal for modification of the New York criteria. Arthritis Rheum 1984:27:361-8.

16 Rudwaleit M, van der Heijde D, Landewé R, et al. The development of Assessment of SpondyloArthritis international Society classification criteria for axial spondyloarthritis (part II): validation and final selection. Ann Rheum Dis 2009;68:777-83.

17 Purcell S, Neale B, Todd-Brown K, et al. PLINK: A tool set for whole-genome association and population-based linkage analyses. Am J Hum Genet 2007;81:559-75.

18 Wang J, Zhuang J, lyer $\mathrm{S}$, et al. Sequence features and chromatin structure around the genomic regions bound by 119 human transcription factors. Genome Res 2012;22:1798-812.

19 Pham D, Vincentz JW, Firulli $A B$, et al. Twist1 regulates Ifng expression in Th1 cells by interfering with Runx3 function. J Immunol 2012;189:832-40.

20 Sanyal A, Lajoie BR, Jain G, et al. The long-range interaction landscape of gene promoters. Nature 2012;489:109-13. 
21 Harmston N, Lenhard B. Chromatin and epigenetic features of long-range gene regulation. Nucleic Acids Res 2013;41:7185-99.

22 Church LD, Filer AD, Hidalgo $E$, et al. Rheumatoid synovial fluid interleukin-17-producing CD4 T cells have abundant tumor necrosis factor-alpha co-expression, but little interleukin-22 and interleukin-23R expression. Arthritis Res Ther 2010;12:R184-R.

23 Yamada H, Nakashima Y, Okazaki K, et al. Th1 but not Th17 cells predominate in the joints of patients with rheumatoid arthritis. Ann Rheum Dis 2008:67:1299-304.

24 Shen H, Goodall JC, Hill Gaston JS. Frequency and phenotype of peripheral blood Th17 cells in ankylosing spondylitis and rheumatoid arthritis. Arthritis Rheum 2009;60:1647-56.

25 Zhang L, Li YG, Li YH, et al. Increased frequencies of Th22 cells as well as Th17 cells in the peripheral blood of patients with ankylosing spondylitis and rheumatoid arthritis. PLOS ONE 2012;7:e31000.

26 Lai NS, Yu HC, Chen HC, et al. Aberrant expression of microRNAs in T cells from patients with ankylosing spondylitis contributes to the immunopathogenesis. Clin Exp Immunol 2013;173:47-57.

27 Seitz M, Lemmel EM, Homfeld J, et al. Enhanced interferon-gamma production by lymphocytes induced by a mitogen from mycoplasma arthritidis in patients with ankylosing spondylitis. Rheumatol Int 1989;9:85-90.
28 Wang C, Liao Q, Hu Y, et al. T lymphocyte subset imbalances in patients contribute to ankylosing spondylitis. Exp Ther Med 2015;9:250-6.

29 Rudwaleit M, Siegert S, Yin Z, et al. Low T cell production of TNF $\alpha$ and IFN $\gamma$ in ankylosing spondylitis: its relation to HLA-B27 and influence of the TNF-308 gene polymorphism. Ann Rheum Dis 2001;60:36-42.

30 Hazlett J, Stamp LK, Merriman T, et al. IL-23R rs11209026 polymorphism modulates IL-17A expression in patients with rheumatoid arthritis. Genes Immun 2012;13:282-7.

31 Sarin R, Wu X, Abraham C. Inflammatory disease protective R381Q IL23 receptor polymorphism results in decreased primary CD4+ and CD8+ human T-cell functional responses. Proc Natl Acad Sci USA 2011;108:9560-5.

32 Coffre $M$, Roumier $M$, Rybczynska M, et al. Combinatorial control of Th17 and Th1 cell functions by genetic variation at genes associated with the IL-23 signaling pathway in spondyloarthritis. Arthritis Rheum 2013;65:1510-21.

33 Ciofani M, Madar A, Galan C, et al. A validated regulatory network for Th17 cell specification. Cell 2012;151:289-303.

34 Cope A, Le Friec G, Cardone J, et al. The Th1 life cycle: molecular control of IFN- $\gamma$ to IL-10 switching. Trends Immunol 2011;32:278-86.

35 Gregory AP, Dendrou CA, Attfield KE, et al. TNF receptor 1 genetic risk mirrors outcome of anti-TNF therapy in multiple sclerosis. Nature 2012;488:508-11. 